\title{
Educação alimentar e nutricional no combate à obesidade infantil: visões do Brasil e do mundo
}

\author{
Food and Nutrition Education in the fight against \\ childhood obesity: Brazilian and international views
}

Mariana Almeida Viveiros de Castro', Grazille Correa de Lima', Gabriella Pinto Belfort ${ }^{2}$

${ }^{\prime} G r a d u a n d a$ do Curso de Nutrição do Centro Universitário IBMR

${ }^{2}$ Doutora, Docente em Nutrição do Centro Universitário IBMR

E-mail para correspondência: Mariana Almeida Viveiros de Castro -

maricotacastro@hotmail.com

\begin{abstract}
Resumo
O objetivo desse trabalho foi realizar uma revisão literária a respeito da importância da educação alimentar e nutricional no combate à obesidade infantil e investigar diferentes práticas em educação com resultados positivos ao redor do mundo. Consideramos os diversos fatores associados a obesidade infantil e a abordagem estratégica e multidisciplinar da educação alimentar e nutricional como forma de promoção de hábitos alimentares saudáveis. Buscaram-se artigos, estudos e documentos oficiais coletados dos bancos de dados da Literatura Latinoamericana e do Caribe de Informação em Ciências da Saúde (LILACS), PubMed, SciELO no período de 2006 a 2020 que abordavam políticas públicas em educação nutricional no combate à obesidade infantil. Foram utilizadas as palavras chaves: educação alimentar e nutricional, educação alimentar, educação nutricional, obesidade infantil. Destacamos os resultados positivos encontrados de práticas em educação alimentar e nutricional no Brasil, Finlândia Japão e Austrália. Evidências demonstraram associação entre conhecimento nutricional com melhores práticas alimentares ressaltando a importância da educação alimentar e nutricional como importante estratégia para a promoção da saúde
\end{abstract}

Palavras-chave: Educação alimentar e nutricional. Educação alimentar. Educação nutricional. Obesidade infantil.

\begin{abstract}
The objective of this work was to conduct a literary review about the importance of nutritional food education in combating childhood obesity and investigate different practices in education with positive results around the world. We consider the various factors associated with childhood obesity and the strategic and multidisciplinary approach to food and nutrition education as a way to promote healthy eating habits. Articles, studies and official documents from databases from the Latin American and Caribbean Literature on Health Sciences Information (LILACS), PubMed, SciELO, from 2006 to 2020 that addressed public policies on nutritional education in the fight against childhood obesity were searched. The keywords were used: food and nutrition education, food education, nutrition education, childhood obesity. We highlight the positive results found
\end{abstract}


from practices in food and nutrition education in Brazil, Finland, Japan and Australia. Evidence showed an association between nutritional knowledge and better eating practices, showing the importance of food and nutrition education as an important strategy for health promotion.

Keywords: Food and nutrition education. Nutrition education. Nutritional education. Child obesity.

\section{INTRODUÇÃO}

A obesidade infantil é um dos maiores desafios mundiais em saúde pública na atualidade, afetando praticamente todos os países do mundo. Nos últimos 40 anos, o número de crianças e adolescentes em idade escolar com obesidade aumentou mais de 10 vezes, passando de 11 milhões para 124 milhões (estimativa de 2016)ํ.

A obesidade está associada a diversos fatores, como o sedentarismo e o padrão alimentar. No Brasil o que temos visto é uma transição alimentar com um aumento de produtos ultraprocessados e a redução de alimentos in natura em decorrência de um marketing massivo de produtos processados além de uma complexidade de fatores sociais e psicológicos ${ }^{2}$.

Crianças com obesidade tem mais probabilidade de permanecerem obesas quando adultos e ainda correm o risco de desenvolverem doenças crônicas não transmissíveis ${ }^{3}$. Diante desse cenário torna-se urgente a adoção de estratégias para deter o avanço da obesidade infantil.

As escolas oferecem uma oportunidade importante para combater a obesidade infantil, melhorando a nutrição de crianças e adolescentes, fornecendo opções saudáveis de alimentos e bebidas, promovendo atividades físicas e fornecendo educação para a saúde1, sendo um local estratégico para a promoção da mesmał .

A Educação Alimentar e Nutricional (EAN) é vista como uma estratégia fundamental para a prevenção e controle de problemas alimentares e nutricionais ${ }^{5}$ que visa promover no indivíduo a prática autônoma e voluntária de hábitos alimentares saudáveis ${ }^{6}$.

O objetivo desse trabalho foi discutir a importância da EAN e identificar políticas públicas em EAN no Brasil e em outros países direcionadas ao combate da obesidade infantil. 


\section{MÉTODOS}

Para a confecção deste artigo, realizou-se uma revisão de literatura científica nacional e internacional, do tipo narrativa, que utilizou a base de dados Literatura Latino-americana e do Caribe de Informação em Ciências da Saúde (LILACS), PubMed, SciELO, a fim de identificar artigos científicos publicados no período de 2006 a 2020.

A busca nas fontes supracitadas foi realizada utilizando como descritores: "educação nutricional e alimentar", "educação nutricional" e "obesidade infantil", e os correspondentes em inglês "food and nutrition education", "nutrition education" e "childhood obesity". Desta forma, foram considerados para o presente estudo artigos publicados na língua inglesa e portuguesa, relacionados com o tema.

As publicações científicas foram pré-selecionadas pelos títulos, os quais deveriam conter como primeiro critério o termo completo (EAN) e/ou referências à educação nutricional ou obesidade infantil.

Para identificar as políticas públicas em EAN no Brasil e no mundo, relacionadas ao combate da obesidade infantil, foram considerados principalmente os artigos sobre estudos de intervenção ou observacionais de estratégias de EAN.

\section{DESENVOLVIMENTO}

\section{Obesidade Infantil}

Considerada como uma epidemia pela Organização Mundial da Saúde (OMS), a Obesidade também afeta as crianças e adolescentes, o que chamamos de obesidade infantil. O número de crianças ao redor do mundo que se tornaram obesas cresceu dez vezes nas últimas quatro décadas ${ }^{7}$.

De acordo com pesquisa realizada no site do Sistema de Vigilância Alimentar e Nutricional (SISVAN) sobre o estado nutricional de crianças de 5 a 10 anos de idade, no ano de 2019 em âmbito nacional, cerca de 10\% das crianças apresentam peso elevado para a idade ${ }^{8}$ e conforme o Atlas da Obesidade Infantil da Organização Mundial de Saúde (OMS), o Brasil terá em 2030 cerca de 22,8\% de crianças entre 5 a 9 anos e $15,7 \%$ de crianças entre 10 a 19 anos com obesidade 9.

A obesidade infantil é o resultado de uma combinação de fatores, desde a exposição a um ambiente que incentiva o aumento de peso e a obesidade, a comportamentos inadequados e a resposta biológica a esse ambiente ${ }^{10}$, como por exemplo, o aumento 
de atividades sedentárias, da propaganda de alimentos ultraprocessados e do consumo de alimentos considerados obesogênicos ${ }^{11}$.

As causas não são apenas individuais ou familiares, mas também ambientais e sociais, e tem como principais desafios o lobby das grandes industriais alimentícias, a falta de vontade política para o enfrentamento da obesidade e a ausência de pressão pela sociedade civil ${ }^{12}$.

Como consequências a obesidade infantil pode levar ao desenvolvimento precoce de doenças crônicas como resistência à insulina, diabetes, hipertensão, doenças cardiovasculares, distúrbios psicológicos e obesidade na vida adulta ${ }^{3}$. Não se trata apenas de um problema crônico isolado numa fase da vida de um indivíduo, mas a exposição a determinados fatores de risco na infância pode comprometer a saúde do adulto $^{13}$.

O combate à obesidade pode trazer benefícios ao sistema de saúde e à economia dos países. Segundo estudo da OMS, os custos com a obesidade em adultos, no mundo todo, em 2014, foram de dois trilhões de dólares ao ano e a prevenção da obesidade infantil é parte importante da estratégia de combate à doença ${ }^{1}$.

Para o combate à obesidade infantil o Ministério da Saúde do Brasil, em sinergia com o programa pelo Fim da Obesidade Infantil da OMS, apresentou em 2019 o programa Crescer Saudável que propõe algumas estratégias fundamentais para serem implementadas, como a regulação da publicidade de produtos ultraprocessados destinados ao público infantil, mudanças na rotulagem de alimentos, a promoção de ambientes alimentares saudáveis, a promoção do aleitamento materno e o desenvolvimento de ações de EAN nas escolas ${ }^{13}$.

A escola é um importante ambiente para a promoção de hábitos alimentares saudáveis e pode ser compreendida como um espaço público que pode ser utilizado em ações no combate à obesidade infantil, através da promoção da atividade física, estruturação de cantinas saudáveis, utilização da comunidade escolar em prol de ações saudáveis e através da educação alimentar nutricional ${ }^{13}$.

A importância da escola no combate à obesidade infantil é sentida pela sua amplitude no que confere quantidade de crianças que ela consegue atingir; crianças que estão em pleno desenvolvimento de seus hábitos alimentares. $\mathrm{O}$ ambiente social escolar também permite o aprendizado e a prática de hábitos saudáveis e a comunidade escolar cria vínculos que reforçam as mensagens de saúde ensinadas na escola. Por 
meio da EAN as escolas conseguem incentivar a alimentação saudável através do currículo e além de tudo há evidências de que ações sistemáticas em escolas com frutas e vegetais aumentam o consumo diário destes por crianças ${ }^{14}$.

\section{Educação Alimentar e Nutricional}

A EAN permite amplas discussões em saúde, por meio do pensamento crítico e reflexivo, colocando significado à alimentação, considerando todas as dimensões que ela atinge ${ }^{13}$. É uma estratégia intersetorial, uma junção de múltiplos saberes, que tem como objetivo construir autonomia no indivíduo para que ele possa voluntariamente adotar hábitos alimentares saudáveis ${ }^{5}$.

O consumo alimentar é um dos fatores que causam o aumento de peso, não apenas pela quantidade de alimentos consumidos, mas também pelo tipo de alimento consumido. A diminuição do consumo dos alimentos in natura e o aumento do consumo de alimentos ultraprocessados contribui para o aumento de peso, já que esses produtos possuem quantidades excessivas de sal e açúcar em sua fabricação e estão associados ao surgimento e crescimento de obesidade e doenças crônicas não transmissíveis ${ }^{15}$. Portanto, para promover hábitos alimentares mais saudáveis e diminuir a obesidade, é importante que as pessoas estejam informadas sobre alimentação e nutrição ${ }^{16}$.

É improvável que intervenções em educação nutricional isoladas resolvam o problema da obesidade infantil. É necessária a interação entre conhecimento e prática, além de ações integradas que envolvam as suas famílias e comunidade, além de políticas públicas que visam a prevenção de doenças ${ }^{15}$. Dessa forma, a educação alimentar e nutricional destaca-se como um campo de conhecimento e de prática contínua e permanente, transdisciplinar, intersetorial e multiprofesional5.

Em 2016, a OMS lançou um relatório em que apresenta recomendações e propõe soluções para o fim da obesidade infantil, e dentre as recomendações propostas destaca-se a Educação Alimentar e Nutricional nos seguintes pontos: incluir EAN no currículo principal das escolas, desenvolver currículos de nutrição e saúde em conjunto entre o setor de educação e setores da saúde, treinar professores na entrega de currículo em educação nutricional, integrar componentes de nutrição e educação em saúde, incluindo habilidades práticas, melhorar a alfabetização e as habilidades nutricionais dos pais e responsáveis e, por último, disponibilizar aulas de preparação de alimentos para crianças, pais e responsáveis ${ }^{17}$. 
Alguns países têm se destacado para promover a alimentação saudável e reduzir a obesidade com ações focadas na educação alimentar e nutricional. Através do banco de dados da World Cancer Research International, atualizado em maio de 2019 tem-se uma ampla visão das ações de políticas governamentais implementadas em todo o mundo e dentre esses países encontra-se o Brasil ${ }^{18}$.

Apresentaremos a seguir como o Brasil tem se destacado em ações de Educação Alimentar e Nutricional e apresentaremos outras interessantes ações como políticas públicas de diferentes países.

\section{Como a Educação Alimentar e Nutricional é aplicada no Brasil}

No Brasil, existem alguns programas e publicações do governo sobre saúde e alimentação, como: Programa Nacional de Alimentação Escolar (PNAE), Programa Nacional de Alimentação e Nutrição (PNAN), Segurança Alimentar e Nutricional (SAN), Guia Alimentar para a População Brasileira, Marco de Referência de Educação Alimentar e Nutricional, Crescer Saudável, entre outras.

Em 2012, a partir da discussão entre os profissionais das áreas de Saúde, Educação e Segurança Alimentar e Nutricional foi compilado um documento, o Marco de Referência de Educação Alimentar e Nutricional, com objetivo aplicar EAN de forma mais assertiva, visto que seu campo de atuação não estava bem definido ${ }^{6}$. As práticas de EAN não vinham tendo resultados consistentes, pois a prevalência de deficiências nutricionais e doenças crônicas não transmissíveis, como a obesidade e doenças cardiovasculares, estavam aumentando e uma das muitas causas seria a alimentação de má qualidade5.

Apesar da estratégia do Marco ser considerada um bom primeiro passo, foi constatado que precisaria de ações, como formação de profissionais que tivessem uma visão integral de toda a EAN 5 . Nesse contexto o Marco propõe a integração de diferentes profissionais para atuar em EAN, contribuindo, assim, para a implementação da lei 11.947 de 2009 que contempla a inclusão da educação alimentar e nutricional no currículo escolar ${ }^{19}$, que segundo as Diretrizes Curriculares nacionais (DCN) de 2013 a educação alimentar e nutricional deve ser tratada de forma universal e integradamente ${ }^{20}$.

O profissional de nutrição, assim como médicos, enfermeiros e odontólogos pensam de forma mais cartesiana, considerando apenas o aspecto fisiológico da alimentação, onde o alimento serve apenas para nutrir, e esquecendo os aspectos 
sociais e culturais ${ }^{21}$. Diante disso questiona-se a cadeira de Pedagogia deve ser incluída, de forma transversal, no curso de nutrição, a fim de melhorar a abordagem de EAN no Brasil ${ }^{22}$.

O Marco de referência de EAN apresenta que são necessários mais recursos educacionais para que seja possível avançar no objetivo de promover a alimentação adequada. Ele não menciona materiais didáticos que possam ser utilizados no processo, visto que a proposta é a constante adaptação dos métodos. Estes precisam de resultados significativos, sempre levando em consideração o teste, na prática, com pessoas reais. As pessoas precisam se sentir em ambiente seguro para poderem se expressar sobre suas preferências alimentares ${ }^{23}$.

A abordagem de EAN no Brasil é intersetorial, buscando sempre a sustentabilidade social, ambiental e econômica. A culinária local é um dos pilares das políticas brasileiras, visto que o país é continental. As políticas sempre incentivam o consumo dos plantios da sua região, com destaque para agricultura familiar local. $\mathrm{O}$ fato de cozinhar seu próprio alimento também é colocado em pauta ${ }^{22}$ deixando claro que o Marco EAN, tem como meta "a promoção do autocuidado e da autonomia e propõe a educação como processo permanente e gerador de autonomia e participação ativa e informada dos sujeitos" 5 .

Em 2019, foi publicado o programa Crescer Saudável, que visa o combate da obesidade na Educação Infantil e Educação Fundamental I, por meio de ações realizadas como ponto focal nas escolas e expandindo para toda comunidade local, buscando sempre englobar todos os aspectos que estão fazendo com que a população infantil esteja se tornando obesa. Este programa é coordenado pelo SUS, buscando a atuação intersetorial, mas principalmente da área de Educação ${ }^{13}$.

O objetivo desse programa é atuar nos ambientes obesogênicos com medidas de implementação de alimentação saudável, atividades físicas regulares, percepção do sono da criança, apoio psicológico das crianças obesas, tudo com a participação da família, pois acredita-se que o ambiente que o indivíduo está inserido tem muita influência nas suas escolhas alimentares ${ }^{13}$.

Algumas das medidas em alimentação do programa seriam a limitação e fiscalização do que se é vendido nas cantinas escolares e seus arredores, sendo proibida a venda de alimentos não saudáveis, tornando assim possível as escolhas saudáveis por parte das crianças. Com o objetivo de ajudar as famílias a terem o acesso mais fácil a frutas, legumes e verduras, as escolas poderiam ajudar a divulgar as feiras, mercados locais 
e sacolões próximos à comunidade em que a escola se encontra. Outra medida para a cooperação da promoção da alimentação saudável seria a confecção de hortas escolares onde os alimentos produzidos nas mesmas podem incrementar as refeições escolares ${ }^{13}$. Como indicado no PNAE, as refeições escolares devem ser preparadas na escola com alimentos in natura ou minimamente processados ${ }^{22}$.

Acredita-se que a escola seja um local apropriado para desenvolver estratégias de promoção à alimentação saudável ${ }^{21}$. Pois é onde a criança passa a maior parte de seu tempo ${ }^{13}$.

Muitas escolas usam uma abordagem expositiva/passiva em que geralmente é utilizado o modelo biomédico reducionista, onde apenas uma pessoa possui o conhecimento e as outras precisam aprender e não tem nada a acrescentar. São feitas palestras e outras apresentações sobre a nutrição e, em alguns casos, a frequência chega a ser de apenas uma vez ao ano ${ }^{22}$. Essa abordagem não vai de encontro com a noção de que educação é uma construção contínua, onde o aprender nunca acaba ${ }^{21}$.

Das escolas públicas brasileiras que utilizam EAN em seu programa escolar, esta geralmente é abordada nas aulas de Ciências e Educação Física, quando também poderia ser abordada nas aulas de História, Geografia e Artes, onde seriam discutidos aspectos socioculturais e ambientais do viés da alimentação. Suas abordagens atuais geralmente são em oficinas de culinária, degustação de alimentos, semana da alimentação saudável e cultivo de horta escolar, nos temas de Higiene dos Alimentos, Alimentação Saudável e Pirâmide Alimentar ${ }^{24}$.

Atualmente busca-se capacitar e educar os professores e a comunidade escolar sobre o tema em EAN, já que crianças são influenciadas pelos adultos que as cercam, principalmente os professores, visto que são os principais responsáveis pelas intervenções $^{22}$. O professor necessita ser adepto de hábitos saudáveis para que seus atos corroborem com seus ensinamentos. Contudo os professores demostram não ter conhecimento para tamanha influência quando se trata de EAN, pois demonstram acreditar que um alimento serve apenas para nutrir, deixando de fora sua parte social e até mesmo cultural ${ }^{21}$, demonstrando que precisamos de uma formação continuada dos educadores infantis pela parte dos profissionais de saúde ${ }^{13}$. Em alguns municípios do país é relatado ter uma reunião por semestre ou até por ano com a nutricionista pela Secretaria de Saúde e os profissionais da área de Educação, onde são discutidos temas de alimentação. Há também uma etapa preparatória onde os professores são capacitados a lecionar sobre grupos de 
nutrientes e suas fontes alimentares, alimentação como promoção de saúde, comida saborosa e nutritiva, entre outros ${ }^{21}$.

\section{EAN sobre a perspectiva de outros países}

Um modelo pioneiro de Educação Alimentar e Nutricional surgiu em 1974 na França. O químico francês Jacques Pursais acreditava que toda criança poderia ser treinada a desenvolver o paladar com aulas que compreendiam os cinco sentidos até a cultura alimentar francesa e a arte do savoir vivre. Com o objetivo de educar pelo gosto, le classes du goût, as aulas elaboradas por Pursais serviram de inspiração para outros países da Europa, como Suécia e Finlândia, a desenvolverem uma educação mais democrática e voltada para a saúde ${ }^{25}$.

A Finlândia pegou o conceito de educar pelos sentidos e desenvolveu o movimento Sapere de educação alimentar, também aplicado em outros países como Suécia e Holanda $^{25}$. De acordo com Sepp e Hojer, o método Sapere trabalha as experiências sensoriais com os alimentos, através dos cinco sentidos e encoraja que as crianças coloquem em palavras o que sentiram na experiência ${ }^{26}$. 0 método preza pelo respeito da individualidade. A criança tem o direito de querer provar ou não, sem julgamentos.

No contexto nórdico a educação nutricional é baseada num processo de aprendizagem democrático. O aluno é estimulado a pensar, criticar, encorajar, refletir e tomar decisões individuais ${ }^{27}$. A proposta de educação pelos sentidos é a mais participativa forma de educação alimentar. O Paladar é uma importante expressão da individualidade da criança. O questionamento do que a criança gosta de comer está de acordo com os seus valores, é uma forma de se afirmar na sociedade ${ }^{27}$. A criança poder discutir com os seus colegas o que ela provou, deixando-a mais consciente e interessada pela comida ${ }^{26}$.

Apesar de ser um método extremamente bem sucedido, o método Sapere apresenta algumas dificuldades. Descrever em palavras experiências novas pode ser difícil tanto para adultos quanto para crianças e as pequenas são muitas vezes influenciadas pela opinião do colega, se uma criança disser que achou o alimento azedo as outras tendem a imitar ${ }^{26}$.

O sucesso do método Sapere, que trabalha com experiências sensoriais deve-se ao fato de que crianças pequenas têm uma maneira natural de explorar o mundo usando os seus sentidos ${ }^{28}$. O alimento é cheio de propriedades e o percebemos através de 
nossos sentidos. As propriedades sensoriais são fatores chaves para a aceitação de frutas, legumes e vegetais na pré-escola ${ }^{29}$.

No Japão, a EAN se tornou parte de uma grande política pública do governo em decorrência da mudança dos hábitos alimentares após a Segunda Guerra Mundial. $\mathrm{O}$ surgimento de novos problemas de saúde associados à má alimentação, como o sobrepeso da população masculina em especial e a obsessão pela magreza exagerada da parte feminina da população, levou o governo criar em 2005 a lei básica de educação alimentar denominada Shokuikuº.

Shokuiku significa educar pela alimentação e sua atuação é bem abrangente, sendo responsáveis pela sua implementação os governos locais, os produtores de alimentos, os educadores e os cidadãos japoneses. Sua aplicação também acontece em múltiplos setores: escolas, centros de saúde e em qualquer empresa ligada a alimentação $0^{30}$. Há um fator multiplicador nessa política pública: professores lecionam e aconselham sobre alimentação nas escolas. Fora das escolas, as crianças dividem o que aprenderam com seus parentes ${ }^{31}$.

No Japão eles adotam uma abordagem que parece mais didática. As informações nutricionais e de saúde sobre os ingredientes incluídos nos almoços são explicitamente ensinadas antes, durante ou após a maioria das refeições ${ }^{32}$. Desde que a lei foi imposta, o número de professores que atuam na educação nutricional passou de 34 para 4.000 em 2015 e em 2014 o governo japonês investiu mais de 200 milhões de ienes na política pública, Shokuiku33.

Programas Nacionais de distribuição de frutas em escolas foram adotados pela Austrália, Reino Unido, Estados Unidos e Nova Zelândia ${ }^{14}$. Nesse contexto, a Austrália merece maior atenção pela estratégia adotada na área de Educação Alimentar e Nutricional. Em 2005, o governo australiano implementou o programa Crunch\&Sip ${ }^{34}$ que determina um tempo em sala de aula, em média 15 minutos, para o consumo de frutas, vegetais e água trazidos de casa pelos alunos. Para o sucesso do programa também foram estabelecidos material escolar de EAN, treinamento de professores e estratégia de comunicação com os pais ${ }^{14}$. 


\section{RESULTADOS DA APLICAÇÃO DE POLÍTICAS PÚBLICAS INTERNACIONAIS DIRECIONADAS AO COMBATE DA OBESIDADE INFANTIL.}

Em artigo publicado no Brasil por Baldasse, Galante e Ganen em 2016 com o objetivo de avaliar os impactos de ações em EAN em uma população escolar de 135 adolescentes, na cidade de São Paulo, teve-se como resultado muitos pontos positivos. Após um ano em intervenções de educação alimentar, 50\% dos estudantes passaram a ler rótulos de alimentos e $63 \%$ passaram a entender as informações contidas nos rótulos. Houve melhoria nas dietas de 33\% dos alunos durante a semana e melhoria de $37 \%$ nas dietas do final de semana. Antes da intervenção, $18 \%$ da amostra se encontrava com obesidade e após a intervenção esse número caiu para $13 \%$. As intervenções realizadas em educação nutricional abrangeram diversos formatos e incluíram jogos, oficinas de culinária e palestras que ajudaram na discussão e reflexão incentivando os alunos a se expressarem sobre os temas ${ }^{35}$.

Estudo feito na Finlândia para avaliar os resultados do método de educação sensorial, também teve bons resultados na disposição das crianças em comerem frutas e legumes e provarem novos alimentos. Após cinco semanas de intervenções sensoriais de educação alimentar mais de 50\% do grupo de controle se demonstrou interessado em comer todas as amostras de alimentos das preparações, um aumento de quase $20 \%$ antes da intervenção ${ }^{36}$. Apesar de haver poucos dados científicos que demonstrem a eficácia do método de educação pelos sentidos, resultados semelhantes foram publicados em outro artigo por Mustonen e Tuorila, em 2009. Neste estudo, foi demonstrada a diminuição da neofobia pelas crianças, o aumento do consumo de frutas e legumes e a melhora das habilidades das crianças em descreverem os aspectos sensoriais e propriedades dos alimentos ${ }^{28}$.

O relatório de 152 auditorias concluídas em 2010 que apresenta a avalição do trabalho nas escolas do oeste australiano denominado de Crush and Sip apresenta interessantes dados. A taxa média do consumo de frutas e legumes foi maior no grupo de menor idade (Jardim de Infância, Pré-Primário e Ano 1): 84\% entre crianças de até 1 ano e 75,8\% entre crianças de 2 a 3 anos, consomem frutas e legumes. Menores taxas de participação estavam entre as idades mais altas, $62 \%$ entre crianças de 6 a 7 anos. Entre todos os entrevistados verificou-se uma forte crença positiva no trabalho realizado e o ponto principal apontado foi a promoção da saúde e sentir satisfação em ver as crianças recebendo frutas e legumes. Um dos pontos que mais contribui para dificuldade na aplicação é a limpeza da bagunça que 
as crianças fazem com as frutas e legumes, demandando tempo e consequente dificuldade em fornecer frutas e legumes para as crianças ${ }^{37}$.

O Japão que possui uma baixa taxa de obesidade infantil na sua população, também apresenta um grande engajamento no aprendizado em educação nutricional. Em um artigo que propõe a análise do ensino de educação nutricional (Shokuiku, em inglês), observa-se que dos 74 entrevistados $68,9 \%$ se interessavam pelo ensino, $94,5 \%$ enxergavam a sua importância, 63,5\% tinham interesse em estudar mais sobre o assunto e $56,8 \%$ pensavam no balanço nutricional de suas refeições ${ }^{38}$.

No quadro 1 está encontra-se um resumo das principais ações em EAN adotadas nos diferentes países que aqui foram citados:

Quadro 1 - Síntese de ações políticas no combate ao excesso de peso durante a infância em nível internacional

\begin{tabular}{|c|c|c|c|}
\hline País, ano & Política & Objetivo & Principais Características \\
\hline $\begin{array}{l}\text { Finlândia, } \\
\text { 200926,27,28,36 }\end{array}$ & Sapere & $\begin{array}{l}\text { Educação } \\
\text { nutricional para } \\
\text { crianças através } \\
\text { de experiências } \\
\text { sensoriais. }\end{array}$ & $\begin{array}{l}\text { Pode ser aplicado em crianças bem } \\
\text { pequenas a partir de } 2 \text { anos. Busca a } \\
\text { percepção do alimento através dos } \\
\text { sentidos e a sua contextualização } \\
\text { através de palavras }\end{array}$ \\
\hline Japão, $2005^{30,31,32,33,38}$ & Shokuiku & $\begin{array}{l}\text { Resgatar a cultura } \\
\text { alimentar } \\
\text { tradicional do } \\
\text { Japão através de } \\
\text { uma dieta } \\
\text { saudável padrão. }\end{array}$ & $\begin{array}{l}\text { Envolve toda uma cadeia responsável } \\
\text { pela alimentação: governo, } \\
\text { educadores, cidadãos e produtores de } \\
\text { alimento. }\end{array}$ \\
\hline Austrália, 200514,34,37 & $\begin{array}{l}\text { Crush and } \\
\text { Sip }\end{array}$ & $\begin{array}{l}\text { Aumentar o } \\
\text { consumo de } \\
\text { frutas e vegetais } \\
\text { através de uma } \\
\text { pausa } \\
\text { exclusivamente } \\
\text { para o seu } \\
\text { consumo, durante } \\
\text { as aulas. }\end{array}$ & $\begin{array}{l}\text { Os próprios alunos levam suas frutas e } \\
\text { legumes de casa o que envolve } \\
\text { também a educação de pais e } \\
\text { professores. }\end{array}$ \\
\hline $\begin{array}{l}\text { Brasil, 2012/20195,6, } \\
13,22,35\end{array}$ & $\begin{array}{l}\text { Marco EAN/ } \\
\text { Crescer } \\
\text { Saudável }\end{array}$ & $\begin{array}{l}\text { Contextualizar a } \\
\text { alimentação } \\
\text { saudável de } \\
\text { acordo com a } \\
\text { realidade do } \\
\text { aluno. }\end{array}$ & $\begin{array}{l}\text { Ressalta a importância do respeito à } \\
\text { cultura alimentar de cada local }\end{array}$ \\
\hline
\end{tabular}

Na última pesquisa realizada pela Organização Mundial de Saúde sobre políticas em nutrição em 2016 e 2017, dos 160 países pesquisados, 89\% implementaram ações em saúde e nutrição nas escolas, e desses, $61 \%$ colocaram educação nutricional no currículo escolar e $29 \%$ colocaram como matéria extracurricular. A educação nutricional nas escolas, em sua maioria, é abordada em lições sobre nutrição e saúde e dietas saudáveis ${ }^{39}$. 
A EAN pode ocorrer, na maioria das vezes, sem se tornar uma modificação do comportamento e controle do paladar infantil. Em alguns casos as crianças são recompensadas com estrelinhas e adesivos por terem incluído alimentos saudáveis nos pratos. Os objetivos de aprendizagem deveriam ser para refletir, criticar e motivar as crianças para que se mantenham informadas de suas escolhas. As crianças devem refletir sobre as suas vivências e estar envolvidas no processo reflexivo do significado e importância do paladar e suas dimensões culturais, sociais e econômicas ${ }^{27}$.

Os estudos sobre EAN demonstram que é um método viável e barato para prevenir doenças e melhorar a saúde e a qualidade da vida dos indivíduos ${ }^{35}$. No entanto, dificilmente atividades isoladas farão surgir algum efeito na prevenção da obesidade infantil, que vem crescendo com passar dos anos. EAN deve ser uma estratégia intersetorial, transdisciplinar de prática contínua e permanente ${ }^{5}$.

\section{CONCLUSÃO}

Foram encontradas diversas práticas em EAN em diferentes países que a adotam como política pública de saúde no combate a obesidade infantil com resultados satisfatórios. No entanto, percebe-se que as práticas encontradas nesta revisão foram todas desenvolvidas todas no âmbito escolar. No Brasil, há a preocupação em colocar a EAN em diversos saberes, respeitando a cultura alimentar de cada região, o saber popular e os diversos significados que a alimentação pode ter. Na Finlândia, a exemplo de outros países nórdicos, EAN é explorada pela educação dos sentidos e de forma participativa estimula o aluno a pensar, criticar, encorajar, refletir e tomar decisões individuais. No Japão, a abordagem em EAN é muito mais didática sendo explicitamente ensinado antes, durante ou após a maioria das refeições e além de tudo envolve uma cadeia de responsáveis pela sua prática que vai além do ambiente escolar. E, por último na Austrália, o envolvimento escolar para que durante as aulas haja uma pausa para o consumo exclusivo de alimentos saudáveis colabora para uma alimentação mais saudável tanto na escola quanto em casa com a expansão desse conhecimento por toda a comunidade escolar.

Dessa forma entendemos a verdade por trás da frase de Nelson Mandela: "A educação é a arma mais poderosa que você pode usar para mudar o mundo" e a Educação alimentar e nutricional tem enorme importância para essa mudança na diminuição da obesidade infantil e da disseminação de hábitos alimentares saudáveis. 


\section{REFERÊNCIAS}

1. WHO, World Health Organization. Taking Action on Childhood Obesity. Geneva: World Health Organization; 2018. (WHO/NMH/PND/ECHO/18.1) Licence: CC BYNCSA 3.0 IGO. Disponível em: https://www.who.int/end-childhoodobesity/publications/taking-action-childhood-obesity-report/en/

2. Brasil - Ministério da Saúde. Secretaria de Atenção à Saúde/Departamento de Atenção Básica. Obesidade: Cadernos de atenção básica - $\mathrm{n}^{0} 12$ série A. Normas e Manuais Técnicos. Brasília (DF): Ministério da Saúde, 2006. Disponível em: http://189.28.128.100/nutricao/docs/geral/doc_obesidade.pdf

3. Bleich SN, Vercammen KA, Zatz LY, et al. Interventions to prevent global childhood overweight and obesity: a systematic review. Lancet Diabetes Endocrinol. 2018 ;6(4):332-346. doi:10.1016/S2213-8587(17)30358-3

4. Schmitz, B. A. S., Recine, E., et al. A escola promovendo hábitos alimentares saudáveis: uma proposta metodológica de capacitação para educadores e donos de cantina escolar. Cad. Saúde Pública [Internet]. 2008 [citado 2020 Abril 11]; 24 (Suppl 2): 312-322. Disponível em:

http://www.scielo.br/scielo.php?script=sci_arttext\&pid=S0102311X2008001400016\&lng=en. https://doi.org/10.1590/S0102311X2008001400016.

5. Bezerra, José Arimatea Barros. Educação alimentar e nutricional: articulação de saberes. Fortaleza: Edições UFC, 2018 [acessado em 2020, março 16]. Disponível em: https://www.fnde.gov.br/index.php/centrais-deconteudos/publicacoes/category/116-alimentacaoescolar?download=12042:educa\%C3\%A7\%C3\%A30-alimentar-nutricionalarticulacao-de-saberes

6. Brasil. Ministério do Desenvolvimento Social e Combate à Fome. Marco de referência de educação alimentar e nutricional para as políticas públicas. Brasília, DF: MDS; Secretaria Nacional de Segurança Alimentar e Nutricional, 2012.

7. NCD Risk Factor Collaboration (NCD-RisC). Worldwide trends in body-mass index, underweight, overweight, and obesity from 1975 to 2016: a pooled analysis of 2416 population-based measurement studies in 128.9 million children, adolescents, and adults. Lancet. 2017;390(10113):2627-2642. doi:10.1016/S0140-6736(17)32129-3

8. Sistema de Vigilância Alimentar e Nutricional. Relatório consolidado de estado nutricional do ano de 2019 [acessado em 21 de abril de 2020]. Disponível em: https://sisaps.saude.gov.br/sisvan/relatoriopublico/index

9. Lobstein, T.; Brinsden, H. Atlas of Childhood Obesity.2019. [acessado em 10 de abril de 2020]. Disponível em: https://www.worldobesity.org/nlsegmentation/global-atlas-on-childhoodobesity

10. WHO, World Health Organization - Report of the commission on ending childhood obesity. Geneva: World Health Organization; 2016 [acessado em 2020, abril 11]. Disponível em: https://apps.who.int/iris/bitstream/handle/10665/204176/9789241510066_eng .pdf;jsessionid=5AF06F2A5393204667EBF5C009D14558? sequence $=1$ 
11. Rede Nacional Primeira Infância. Mapeamento da Ação Finalística "Criança com Saúde" Obesidade na Primeira Infância. 2014 [acessado em 2020, abril 23].

Disponivel em: www.primeirainfancia.org.br

12. Castro, I. R. R. Obesidade: urge fazer avançar políticas públicas para sua prevenção e controle. Cad. Saúde Pública [Internet]. 2017 [citado 2020 abril 9]; 33 (7): e00100017. Disponível em:

http://www.scielo.br/scielo.php?script=sci_arttext\&pid=S0102311X2017000700201\&lng=pt. http://dx.doi.org/10.1590/0102-311x00100017

13. Brasil. Ministério da Educação. Manual instrutivo do programa de prevenção, controle e tratamento da obesidade infantil. Programa Crescer Saudável. 2019.

14. Nathan, N; Wolfenden L.; Butler, M; et al. Vegetable and fruit breaks in Australian primary schools: prevalence, attitudes, barriers and implementation strategies. Health Educ Res. 2011; 26, (4): 722-731. doi:10.1093/her/cyr033

15. Brasil. Ministério da Saúde. Guia Alimentar para a população brasileira.2a ed. Brasília, DF. Secretaria de Atenção à Saúde Departamento de Atenção Básica, 2014 .

16. Triches R. M., Giugliani E. R. J. Obesidade, práticas alimentares e conhecimentos de nutrição em escolares. Rev. Saúde Pública [Internet]. 2005 [citado 2020 março 26]; 39 (4): 541-547. Disponível em: http://www.scielo.br/scielo.php?script=sci_arttext\&pid=S003489102005000400004\&lng=en. http://dx.doi.org/10.1590/S003489102005000400004 .

17. WHO, World Health Organization. Report of the Commission on Ending Childhood Obesity. Implementation plan: executive summary. Geneva. World Health Organization. 2017. (WHO/NMH/PND/ECHO/17.1). Licence: CC BY-NC-SA 3.0 IGO.

18. World Cancer Research Fund International. Noushoring Framework. 2019 [acessado em 2020, abril 10]. Disponível em:

https://www.wcrf.org/sites/default/files/10_Give_nutrition_education_and_s kills.pdf

19. Brasil. Lei n.11.947 de 16 de junho de 2009 - Dispõem sobre o atendimento da alimentação escolar. Disponível em http://www.planalto.gov.br/ccivil_03/_Ato2007-2010/2009/Lei/L11947.htm

20. Brasil. Ministério da Educação. Secretária de Educação Básica. Diretizes Curriculares Nacionais da Educação Básica. Brasilia. DF: Ministério da Educação, 2013.

21. Magalhães, H. H. S. R., Porte, L. H. M. Percepção de educadores infantis sobre educação alimentar e nutricional. Ciência \& Educação (Bauru). 2019 [citado 2020, março 10]. 25 (1), 131-144. Disponível em: http://www.scielo.br/scielo.php?script=sci_arttext\&pid=S151673132019000100131\&lng=en\&nrm=iso .https://doi.org/10.1590/1516731320190010009

22. Ottoni, I. C.; Domene, S. M. A.; Bandoni, D. H. Educação alimentar e nutricional em escolas: uma visão do Brasil. DEMETRA: Alimentação, Nutrição \& Saúde, 2019. [citado 2020, março 10] 14, p. e38748. Disponível em: https://www.epublicacoes.uerj.br/index.php/demetra/article/view/38748.

doi:https://doi.org/10.12957/demetra.2019.38748 
23. Macedo, I., \& Aquino, R. O. Marco de referência de educação alimentar e nutricional para políticas públicas no Brasil no contexto do atendimento nutricional. DEMETRA: Alimentação, Nutrição \& Saúde. 2018. [citado 2020, março 9] 13(1), 21-35. Disponível em: https://www.epublicacoes.uerj.br/index.php/demetra/article/view/28663 https://doi.org/10.12957/demetra.2018.28663

24. Batista, M. da S. A., Mondini L., Jaime, P. C. Ações do Programa Saúde na Escola e da alimentação escolar na prevenção do excesso de peso infantil: experiência no município de Itapevi, São Paulo, Brasil, 2014. Epidemiol. Serv. Saúde [Internet]. 2017 [citado 2020 março 11]; 26 (3): 569-578. Disponível em: http://www.scielo.br/scielo.php?script=sci_arttext\&pid=S223796222017000300569\&lng=en. http://dx.doi.org/10.5123/s167949742017000300014.

25. Wilson, B. Como aprendemos a comer: por que a alimentação dá tão errado para tanta gente e como fazer escolas melhores. Rio de Janeiro: Zahar, 2017.

26. Sepp, H. and Höijer, K. Food as a tool for learning in everyday activities at preschool na exploratory study from Sweden. Food \& Nutrition Research. 2016; 60 (1) 32603. https://doi.org/10.3402/fnr.v60.32603

27. Leer, J., and K. Wistoft. Taste in Food Education: A Critical Review Essay. Food and Foodways. 2017 [citado 2020, abril 9]; 26 (4): 329-349. Disponível em: https://scholar.google.com/scholar_lookup?hl=en\&publication_year=2018\&pa ges $=329-$ 349\&issue $=4$ \&author $=$ J.+Leer\&author $=$ K. + Wistoft\&title $=$ Taste + in + Food + Educa tion\%3A+A+Critical+Review+Essay doi:10.1080/07409710.2018.1534047.

28. Mustonen, S., Tuorila, H. Sensory education decreases food neophobia score and encourages trying unfamiliar foods in 8-12-year-old children. Elsevier: Food Quality and Preference. 2009; 21 (4): 353-360. https://doi.org/10.1016/j.foodqual.2009.09.001

29. Sandell, Mikkelsen, Lyytikainen, et al. The future for food education of children. Elsevier: Futures. 2016. 83: (Futures for Food) 15-23. https://doi.org/10.1016/j.futures.2016.04.006

30. Mah, C. L. Shokuiku: Governing Food and Public Health in Contemporary Japan. Journal of Sociology, 2010; 46 (4): 393-412. doi:10.1177/1440783310384455.

31. Nakamura T. The integration of school nutrition program into health promotion and prevention of lifestyle-related diseases in Japan. Asia Pac J Clin Nutr. 2008; [citado 2020, abril 10]; 17 (1): 349-351. PMID: 18296376.

32. Moffata, T., Thrasher, D. School meal programs and their potential to operate as school-based obesity prevention and nutrition interventions: case studies from France and Japan. Critical Public Health. 2014 [citado 2020, abril 11]; 26 (2): 133146. https://doi.org/10.1080/09581596.2014.957654.

33. Nerman, D. Food Education the law in Japan. CBC News. Canadá: 2015 Janeiro, 12 [2015 Janeiro, 12; 2020 abril 23]. Disponível em:

https://www.cbc.ca/news/health/food-education-the-law-in-japan-1.2894279

34. Crunch \& Sip. Australia. Acessado em 19 de abril de 2020. Disponível em: https://www.crunchandsip.com.au/

35. Baldasso J. G., Galante A. P., Ganen A. P. Impact of actions of food and nutrition education program in a population of adolescents. Rev. Nutr. [Internet]. 2016 
[citado 2020 março 11]; 29 (1): 65-75. Disponível em: http://www.scielo.br/scielo.php?script=sci_arttext\&pid=S141552732016000100065\&lng=en. https://doi.org/10.1590/167898652016000100007.

36. Hoppu, Prinz et al. Impact of sensory-based food education in kindergarten on willingness to eat vegetables and berries. Food \& Nutrition Research. 2015 [citado 2020, março 9]. 59: 10.3402/fnr.v59.28795; https://doi.org/10.3402/fnr.v59.28795

37. Barnes, R. Crunch\&Sip ${ }^{\circledR}$ Policy Evaluation Results of the Audit Survey and Tally Charts 2010; 2010 [acesso em 19 de abril de 2020]. Disponível em:

https://www.crunchandsip.com.au/assets/downloads/2012-04-10-crunchsipaudit-report.pdf

38. Kanda, S., Chino, K. et al. The educational effect of shokuiku (food and nutrition education) taught in English as a form internacional exchange in Japanese elementar schools. Jpn J Health \& Human Ecology. 2012. 78 (4): 91-102; https://doi.org/10.3861/jshhe.78.91

39. WHO, World Health Organization. Global nutrition policy review 2016-2017: country progress in creating enabling policy environments for promoting healthy diets and nutrition. Geneva: World Health Organization; 2018. Licence: CC BY-NC-SA 3.0 IGO.

Submissão: 01/07/2020

Aprovação: 08/06/2021 